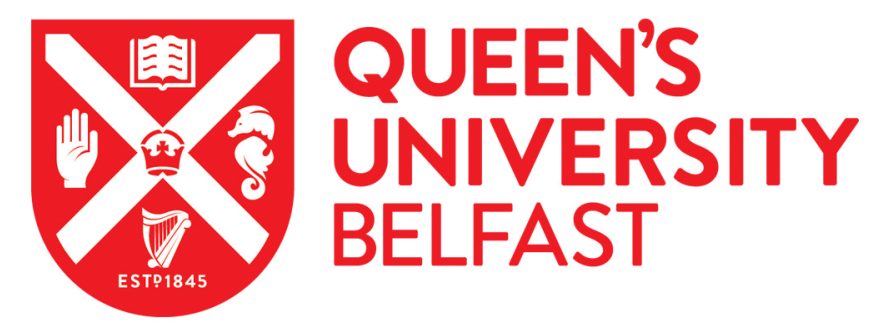

\title{
SN2018kzr: A Rapidly Declining Transient from the Destruction of a White Dwarf
}

McBrien, O. R., Smartt, S. J., Chen, T. W., Inserra, C., Gillanders, J. H., Sim, S. A., Jerkstrand, A., Rest, A., Valenti, S., Roy, R., Gromadzki, M., Taubenberger, S., Flörs, A., Huber, M. E., Chambers, K. C., Gal-Yam, A., Young, D. R., Nicholl, M., Kankare, E., ... Rau, A. (2019). SN2018kzr: A Rapidly Declining Transient from the Destruction of a White Dwarf. Astrophysical Journal Letters, 885(1), [L23]. https://doi.org/10.3847/20418213/ab4dae

Published in:

Astrophysical Journal Letters

Document Version:

Publisher's PDF, also known as Version of record

Queen's University Belfast - Research Portal:

Link to publication record in Queen's University Belfast Research Portal

Publisher rights

(C) 2019. The American Astronomical Society. All rights reserved. This work is made available online in accordance with the publisher's policies. Please refer to any applicable terms of use of the publisher.

\section{General rights}

Copyright for the publications made accessible via the Queen's University Belfast Research Portal is retained by the author(s) and / or other copyright owners and it is a condition of accessing these publications that users recognise and abide by the legal requirements associated with these rights.

Take down policy

The Research Portal is Queen's institutional repository that provides access to Queen's research output. Every effort has been made to ensure that content in the Research Portal does not infringe any person's rights, or applicable UK laws. If you discover content in the Research Portal that you believe breaches copyright or violates any law, please contact openaccess@qub.ac.uk. 


\title{
SN2018kzr: A Rapidly Declining Transient from the Destruction of a White Dwarf
}


Anders Jerkstrand $^{4}(\mathbb{D})$, Armin Rest ${ }^{5}$, Stefano Valenti ${ }^{6}$ (D) Rupak Roy $^{7}$ (1), Mariusz Gromadzki ${ }^{8}$, Stefan Taubenberger ${ }^{4}$ (1),

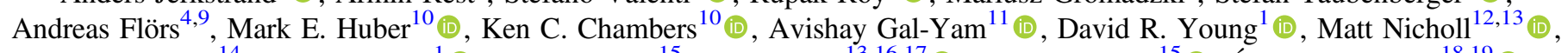

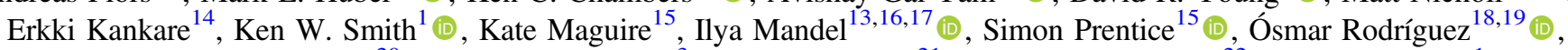
Jonathan Pineda Garcia ${ }^{20}$, Claudia P. Gutiérrez ${ }^{3}\left(\mathbb{C}^{\circ}\right.$, Lluís Galbany ${ }^{21}{ }^{10}$, Cristina Barbarino ${ }^{22}$, Peter S. J. Clark ${ }^{1}$, Jesper Sollerman ${ }^{22}$ (1), Shrinivas R. Kulkarni ${ }^{23}$ (1), Kishalay De ${ }^{23}$, David A. H. Buckley ${ }^{24}$, and Arne Rau ${ }^{2}$ (1)

1 Astrophysics Research Centre, School of Mathematics and Physics, Queen's University Belfast, BT7 1NN, UK; omcbrien02@qub.ac.uk

${ }^{2}$ Max-Planck-Insitut für Extraterrestrische Physik, Giessenbachstraße, D-85748, Garching bei München, Germany

${ }^{3}$ Department of Physics and Astronomy, University of Southampton, Southampton SO17 1BJ, UK

${ }^{4}$ Max-Planck-Institut für Astrophysik, Karl-Schwarzschild-Straße 1, D-85748 Garching bei München, Germany ${ }^{5}$ Space Telescope Science Institute, 3700 San Martin Drive, Baltimore, MD 21218, USA

${ }^{6}$ Department of Physics, University of California, 1 Shields Avenue, Davis, CA 95616-5270, USA

${ }^{7}$ Inter-University Centre for Astronomy and Astrophysics, Ganeshkhind, Pune 411007, Maharashtra, India

${ }^{8}$ Warsaw University Astronomical Observatory, Al. Ujazdowskie 4, 00-478, Warszawa, Poland

${ }^{9}$ European Southern Observatory, Karl-Schwarzschild-Straße 2, D-85748 Garching bei München, Germany

${ }^{10}$ Institute of Astronomy, University of Hawaii, 2680 Woodlawn Drive, Honolulu, HI 96822, USA

11 Department of Particle Physics and Astrophysics, Weizmann Institute of Science, Rehovot 76100, Israel

${ }^{12}$ Institute for Astronomy, University of Edinburgh, Royal Observatory, Blackford Hill, EH9 3HJ, UK

${ }^{13}$ Institute for Gravitational Wave Astronomy, School of Physics and Astronomy, University of Birmingham, Birmingham B15 2TT, UK

${ }^{14}$ Tuorla Observatory, Department of Physics and Astronomy, University of Turku, FI-20014 Turku, Finland

${ }^{15}$ School of Physics, Trinity College Dublin, The University of Dublin, Dublin 2, Ireland

${ }^{16}$ Monash Centre for Astrophysics, School of Physics and Astronomy, Monash University, Clayton, Victoria 3800, Australia

${ }^{17}$ OzGrav, Australian Research Council Centre of Excellence for Gravitational Wave Discovery, Australia

${ }^{18}$ Departamento de Ciencias Fisicas, Universidad Andres Bello, Avda. Republica 252, Santiago, Chile

${ }^{19}$ Millennium Institute of Astrophysics (MAS), Nuncio Monseñor Sótero Sanz 100, Providencia, Santiago, Chile

${ }^{20}$ Departamento de Astronomía, Universidad de Chile, Camino El Observatorio 1515, Santiago, Chile

${ }^{21}$ Departamento de Física Teórica y del Cosmos, Universidad de Granada, E-18071 Granada, Spain

${ }^{23}$ Cahill Centre for Astrophysics, California Institute of Technology, 1200 East California Boulevard, Pasadena, CA 91125, USA

${ }^{24}$ South African Astronomical Observatory, PO Box 9, Observatory 7935, Cape Town, South Africa

Received 2019 September 6; revised 2019 October 7; accepted 2019 October 15; published 2019 October 31

\begin{abstract}
We present SN2018kzr, the fastest declining supernova-like transient, second only to the kilonova, AT2017gfo. SN2018kzr is characterized by a peak magnitude of $M_{r}=-17.98$, a peak bolometric luminosity of $\sim 1.4 \times 10^{43} \mathrm{erg} \mathrm{s}^{-1}$, and a rapid decline rate of $0.48 \pm 0.03 \mathrm{mag} \mathrm{day}^{-1}$ in the $r$ band. The bolometric luminosity evolves too quickly to be explained by pure ${ }^{56} \mathrm{Ni}$ heating, necessitating the inclusion of an alternative powering source. Incorporating the spin-down of a magnetized neutron star adequately describes the lightcurve and we estimate a small ejecta mass of $M_{\mathrm{ej}}=0.10 \pm 0.05 M_{\odot}$. Our spectral modeling suggests the ejecta is composed of intermediate mass elements including $\mathrm{O}, \mathrm{Si}$, and $\mathrm{Mg}$ and trace amounts of Fe-peak elements, which disfavors a binary neutron star merger. We discuss three explosion scenarios for SN2018kzr, given the low ejecta mass, intermediate mass element composition, and high likelihood of additional powering - the core collapse of an ultrastripped progenitor, the accretion induced collapse (AIC) of a white dwarf, and the merger of a white dwarf and neutron star. The requirement for an alternative input energy source favors either the AIC with magnetar powering or a white dwarf-neutron star merger with energy from disk wind shocks.
\end{abstract}

Unified Astronomy Thesaurus concepts: Magnetars (992); Supernovae (1668); White dwarf stars (1799)

\section{Introduction}

Within the already diverse range of explosive transients known to exist is a subset of rapidly evolving objects commonly referred to as fast transients. The definition of "fast" has changed over time as more of these objects have been discovered, but in general they display rise and fall times much shorter than for a typical supernova, appearing and fading from view in a matter of weeks. Naturally, to exhibit a more rapid evolution than a typical supernova, a different explosion scenario is needed to explain the event beyond the standard single progenitor scenarios studied. Examples of fast transients include Type Iax objects such as SN2002cx
(Li et al. 2003), .Ia candidates including SN2002bj and SN2010X (Kasliwal et al. 2010; Poznanski et al. 2010), Ca-rich Type I supernovae like SN2005E (Perets et al. 2010; Valenti et al. 2014), and other fast fading transients interesting in their own right like SN2005ek (Drout et al. 2013), iPTF14gqr (De et al. 2018), iPTF16asu (Whitesides et al. 2017), and KSN2015K (Rest et al. 2018). Events such as these tend to be rarer in occurrence, making up only a small fraction of the normal supernova rate. The fastest transients yet have been discovered in recent years, with the best examples being those of AT2018cow (Prentice et al. 2018) and the more recently discovered SN2019bkc (Chen et al. 2019). The fastest optical transient known is, of course, the kilonova AT2017gfo 
(Abbott et al. 2017; Andreoni et al. 2017; Arcavi et al. 2017; Chornock et al. 2017; Coulter et al. 2017; Cowperthwaite et al. 2017; Drout et al. 2017; Evans et al. 2017; Kasliwal et al. 2017; Lipunov et al. 2017; Nicholl et al. 2017; Pian et al. 2017; Smartt et al. 2017; Tanvir et al. 2017; Troja et al. 2017; Utsumi et al. 2017; Valenti et al. 2017), the result of the radioactive decay of heavy $r$-process elements synthesized in the merger of two neutron stars (Kasen et al. 2017; Metzger 2017). Modern survey telescopes, with their nightly cadences and wide fields of view, are uncovering an increasing number of fast transients like these every year.

Here we report photometric and spectroscopic observations of SN2018kzr, which was independently discovered by the ATLAS survey (Tonry et al. 2018) and the Zwicky Transient Facility (ZTF; Bellm et al. 2019), along with modeling of the bolometric lightcurve and early spectra, and a discussion of plausible explosion scenarios of this object. Throughout this Letter we adopt cosmology of $H_{0}=70 \mathrm{~km} \mathrm{~s}^{-1} \mathrm{Mpc}^{-1}$, $\Omega_{m}=0.3$, and $\Omega_{\Lambda}=0.7$ and assume a foreground reddening of $A_{V}=0.113$ (NED) alongside the Cardelli et al. (1989) extinction law with $R_{V}=3.1$. All phases are measured with respect to the ZTF discovery epoch, MJD 58480.422, unless otherwise stated.

\section{Observations}

\subsection{Discovery}

SN2018kzr was independently discovered by both ZTF (as ZTF18adaykvg) and ATLAS (as ATLAS18bchu) within $2 \mathrm{hr}$ of each other on the night of 2018 December 28. ZTF discovered it on MJD 58480.422 at $r=18.58 \pm 0.11$ (Fremling 2018) and it was ingested into the public alerts broker LASAIR (Smith et al. 2019), while ATLAS detected it in a $30 \mathrm{~s}$ image on MJD 58480.499 with magnitude $o=$ $18.75 \pm 0.14$. ATLAS has the closest nondetection in time, with four images taken at a midpoint of MJD 58478.520 $(-1.902$ days $)$ and a combined $3 \sigma$ upper limit of $o>19.66$. The rapid rise triggered an ePESSTO (Smartt et al. 2015) classification spectrum on MJD $58482.317(+1.895$ days) and again on MJD 58483.247 (+2.825 days, Pineda et al. 2018; Razza et al. 2018), which suggested a preliminary Type Ic classification. It is coincident ( $0 . " 6$ offset) with the blue, $g=20.5$, galaxy SDSS J082853.50+010638.6.

\subsection{Photometry}

Observations were made over a period of two weeks, during which time the transient faded rapidly (see Table 1). Groundbased grizJHK photometry was collected as part of the GREAT survey (Chen et al. 2018) using GROND (Greiner et al. 2008), along with griz photometry from the Liverpool Telescope (LT) and gri photometry from the New Technology Telescope (NTT). As the transient faded rapidly and was coincident with its host galaxy (Figure 1), difference imaging was essential for all epochs which we carried out using HOTPANTS (Becker 2015). The reference epochs used for the GROND, LT, and NTT images are listed in Table 1. Photometry was measured with point-spread-function fitting on the difference images, with the image zero-points set from Pan-STARRS1 reference stars in the field (Chambers et al. 2016; Magnier et al. 2016).

Eight epochs of UV imaging were taken with Swift. Due to its fast fading, it was only recovered in four epochs in $U V W 2$, and three in $U V M 2$ and $U V W 1$. The Swift data are presented in Table 2. These magnitudes have not been host subtracted as host contributions were negligible in the exposures.

A strikingly rapid decline was measured across all the griz bands at rates $\Delta g=0.48 \pm 0.03 \mathrm{mag} \mathrm{d}^{-1}, \Delta r=0.48 \pm$ $0.03 \mathrm{mag} \mathrm{d}^{-1}, \Delta i=0.54 \pm 0.04 \mathrm{mag} \mathrm{day}^{-1}, \Delta z=0.39 \pm$ 0.04 mag day $^{-1}$, all measured over the nine night period for which GROND was observing. This is faster than SN2019bkc, declining at a rate of $\Delta r=0.41 \pm 0.01 \mathrm{mag} \mathrm{day}^{-1}$ (Chen et al. 2019), which had been the fastest declining supernova-like transient until now. The red bands ( $i$ and $z$ ) are similar to the kilonova AT2017gfo (see Figure 2). There appears to be no significant near-infrared flux in the GROND $J H K$ images after image subtraction so we do not consider them further here.

\subsection{Spectroscopy}

A total of 12 spectra were taken beginning on MJD $58482.317(+1.895$ days $)$ with the aforementioned initial NTT:EFOSC2 classification spectrum from ePESSTO. A second NTT:EFOSC2 spectrum was taken on MJD $58483.247(+2.825$ days) along with a third NTT:EFOSC2 spectrum on MJD $58484.172(+3.750$ days $)$ with a broader wavelength coverage (3400-10300 $\AA$ as opposed to the former $3700-9300 \AA$ ). On MJD 58487, three optical to near-infrared spectra were taken by SALT:RSS, VLT:Xshooter, and Gemini:GMOS-N, along with a Keck:LRIS spectrum on the subsequent night. A second Gemini: GMOS-N spectrum was taken on MJD $58489.437(+9.015$ days). Another NTT:EFOSC2 spectrum was obtained on MJD 58490.316 (+9.894 days) but was of a very low signal-to-noise, showing no identifiable emission or absorption features. One more Keck:LRIS spectrum was taken on MJD 58494.356 $(+14.136$ days $)$ showing faint emission most notably around $8500 \AA$. The final spectrum taken was a VLT:Xshooter spectrum from MJD 58525.119 (+44.697 days) which showed narrow nebular emission lines from the host galaxy but no detectable flux from SN2018kzr. The [O II] doublet $\lambda \lambda 3726.03,3926.47$ was resolved into two components and a double Gaussian with full width at half maximum FWHM $=1.7 \AA$ was fit to the profiles. The [O III] $\lambda 5006.84$ line was also detected and the mean of all three centroids gave $z=0.05298 \pm 0.00005$. For the cosmology we adopt, this equates to a luminosity distance of $236 \mathrm{Mpc}$. This VLT:Xshooter spectrum was also used to subtract host continuum flux from the later-time spectra (those from MJD 58487 onward).

\section{Data Analysis \\ 3.1. Lightcurve Modeling and Comparison}

Using the griz photometry (Table 1, Figure 2), a bolometric lightcurve was constructed with SUPEROL (Nicholl 2018), which integrates under blackbody fits to the spectral energy distribution estimated at each epoch of observation (Figure 3). Based on the Arnett formalism, we may constrain the ejecta mass expected from the opacity, photospheric velocity of the ejecta, and an estimate of the rise time of the bolometric lightcurve. Supposing an opacity of $0.1-0.2 \mathrm{~cm}^{2} \mathrm{~g}^{-1}$, velocity of the order of $10^{4} \mathrm{~km} \mathrm{~s}^{-1}$ and a rise time $<3$ days, we anticipate an ejecta mass $\lesssim 0.1 M_{\odot}$.

For parameter estimation we have fitted two different powering models, and a combination of both, to the bolometric lightcurve using the formalism and methods described in Inserra et al. (2013). The powering sources were ${ }^{56} \mathrm{Ni}$ 
Table 1

The griz Photometric Log of SN2018kzr

\begin{tabular}{|c|c|c|c|c|c|c|c|}
\hline Date & MJD & Phase & $g$ & $r$ & $i$ & $z$ & Instrument \\
\hline 2018 Dec 23 12:23:02 & 58475.516 & -4.906 & $>19.0$ & $\ldots$ & $\ldots$ & $\ldots$ & ZTF \\
\hline 2018 Dec 24 12:11:31 & 58476.508 & -3.914 & $\ldots$ & $>18.90^{\mathrm{a}}$ & $\cdots$ & $\cdots$ & ATLAS \\
\hline 2018 Dec 26 12:28:48 & 58478.520 & -1.902 & $\cdots$ & $>19.80^{\mathrm{a}}$ & $\cdots$ & $\cdots$ & ATLAS \\
\hline 2018 Dec 28 10:07:48 & 58480.422 & 0.000 & $\cdots$ & $18.58 \pm 0.11$ & $\cdots$ & $\cdots$ & ZTF \\
\hline 2018 Dec 28 10:34:01 & 58480.440 & 0.018 & $\ldots$ & $18.54 \pm 0.11$ & $\cdots$ & $\cdots$ & ZTF \\
\hline 2018 Dec 28 11:26:59 & 58480.477 & 0.055 & $18.14 \pm 0.08$ & $\cdots$ & $\cdots$ & $\cdots$ & ZTF \\
\hline 2018 Dec 28 11:36:22 & 58480.484 & 0.062 & $18.25 \pm 0.09$ & $\ldots$ & $\ldots$ & $\ldots$ & ZTF \\
\hline 2018 Dec 28 11:58:04 & 58480.499 & 0.077 & $\ldots$ & $18.75 \pm 0.14^{\mathrm{a}}$ & $\cdots$ & $\cdots$ & ATLAS \\
\hline 2018 Dec 28 12:11:05 & 58480.508 & 0.086 & $\cdots$ & $18.62 \pm 0.13^{\mathrm{a}}$ & $\cdots$ & $\cdots$ & ATLAS \\
\hline 2018 Dec 28 12:25:10 & 58480.517 & 0.095 & $\cdots$ & $18.76 \pm 0.14^{\mathrm{a}}$ & $\cdots$ & $\cdots$ & ATLAS \\
\hline 2018 Dec 28 12:38:46 & 58480.527 & 0.105 & $\cdots$ & $18.52 \pm 0.11^{\mathrm{a}}$ & $\cdots$ & $\cdots$ & ATLAS \\
\hline 2018 Dec 29 10:24:06 & 58481.433 & 1.011 & $\cdots$ & $18.54 \pm 0.08$ & $\cdots$ & $\cdots$ & ZTF \\
\hline 2018 Dec 29 11:28:27 & 58481.478 & 1.056 & $\cdots$ & $18.62 \pm 0.11$ & $\cdots$ & $\cdots$ & ZTF \\
\hline 2018 Dec 30 12:02:52 & 58482.502 & 2.080 & $\ldots$ & $18.68 \pm 0.16^{\mathrm{a}}$ & $\cdots$ & $\cdots$ & ATLAS \\
\hline 2018 Dec 30 12:15:50 & 58482.511 & 2.089 & $\cdots$ & $18.58 \pm 0.66^{\mathrm{a}}$ & $\cdots$ & $\cdots$ & ATLAS \\
\hline 2018 Dec 30 12:21:36 & 58482.515 & 2.093 & $\cdots$ & $17.83 \pm 0.55^{\mathrm{a}}$ & $\cdots$ & $\cdots$ & ATLAS \\
\hline 2018 Dec 30 12:40:19 & 58482.528 & 2.106 & $\cdots$ & $18.70 \pm 0.35^{\mathrm{a}}$ & $\cdots$ & $\cdots$ & ATLAS \\
\hline 2018 Dec 31 06:34:28 & 58483.274 & 2.852 & $18.83 \pm 0.01^{\mathrm{b}}$ & $18.88 \pm 0.01^{\mathrm{b}}$ & $19.07 \pm 0.02^{\mathrm{b}}$ & $19.08 \pm 0.04^{\mathrm{b}}$ & GROND \\
\hline 2019 Jan 1 03:40:23 & 58484.153 & 3.731 & $19.66 \pm 0.09$ & $19.33 \pm 0.05$ & $19.46 \pm 0.02$ & $19.62 \pm 0.02$ & GROND \\
\hline 2019 Jan 1 04:19:27 & 58484.180 & 3.758 & $18.92 \pm 0.03$ & $18.68 \pm 0.04$ & $18.43 \pm 0.03$ & $\cdots$ & LCOGT \\
\hline 2019 Jan 2 00:33:32 & 58485.023 & 4.601 & $20.49 \pm 0.06$ & $19.75 \pm 0.12$ & $19.74 \pm 0.10$ & $20.06 \pm 0.16$ & IO:O \\
\hline 2019 Jan 3 05:32:53 & 58486.231 & 5.809 & $21.37 \pm 0.03$ & $20.52 \pm 0.01$ & $20.58 \pm 0.03$ & $20.28 \pm 0.02$ & GROND \\
\hline 2019 Jan 3 09:10:04 & 58486.382 & 5.960 & $\cdots$ & $20.34 \pm 0.22$ & $\cdots$ & $\cdots$ & P60 \\
\hline 2019 Jan 3 12:04:56 & 58486.503 & 6.081 & $\ldots$ & $>20.51^{\mathrm{a}}$ & $\cdots$ & $\cdots$ & ATLAS \\
\hline 2019 Jan 4 00:51:08 & 58487.036 & 6.614 & $22.02 \pm 0.06$ & $20.98 \pm 0.19$ & $20.96 \pm 0.06$ & $20.88 \pm 0.10$ & IO:O \\
\hline 2019 Jan 4 07:42:01 & 58487.321 & 6.899 & $22.04 \pm 0.05$ & $21.41 \pm 0.02$ & $21.35 \pm 0.02$ & $20.96 \pm 0.02$ & GROND \\
\hline 2019 Jan 5 01:04:36 & 58488.045 & 7.623 & $22.24 \pm 0.09$ & $21.78 \pm 0.17$ & $21.53 \pm 0.11$ & $20.93 \pm 0.20$ & IO:O \\
\hline 2019 Jan 5 07:56:24 & 58488.331 & 7.909 & $22.72 \pm 0.05$ & $22.33 \pm 0.03$ & $22.25 \pm 0.06$ & $21.63 \pm 0.04$ & GROND \\
\hline 2019 Jan 6 00:17:30 & 58489.012 & 8.590 & $22.82 \pm 0.09$ & $22.11 \pm 0.20$ & $21.87 \pm 0.11$ & $21.28 \pm 0.12$ & IO:O \\
\hline 2019 Jan 7 07:09:55 & 58490.299 & 9.877 & $22.91 \pm 0.03$ & $22.72 \pm 0.06$ & $22.98 \pm 0.08$ & $21.98 \pm 0.06$ & GROND \\
\hline 2019 Jan 8 07:21:57 & 58491.307 & 10.885 & $23.14 \pm 0.05$ & $22.94 \pm 0.05$ & $22.61 \pm 0.07$ & $22.06 \pm 0.05$ & GROND \\
\hline 2019 Jan 9 07:10:43 & 58492.299 & 11.877 & $23.32 \pm 0.07$ & $23.25 \pm 0.07$ & $\cdots$ & $>21.84$ & GROND \\
\hline 2019 Jan 14 05:08:34 & 58497.214 & 16.792 & $23.74 \pm 0.07$ & $23.83 \pm 0.08$ & $23.20 \pm 0.05$ & $\cdots$ & EFOSC2 \\
\hline 2019 Jan 27 07:11:23 & 58510.300 & 29.878 & $>24.44$ & $>24.94$ & $>24.11$ & $\cdots$ & EFOSC2 \\
\hline 2019 Feb 5 03:08:28 & 58519.131 & 38.709 & ref & ref & ref & ref & GROND \\
\hline 2019 Mar 7 02:20:35 & 58549.098 & 68.676 & ref & ref & ref & $\cdots$ & EFOSC2 \\
\hline 2019 Apr 26 20:27:41 & 58599.853 & 119.431 & ref & ref & ref & ref & IO:O \\
\hline SDSS DR15 & Host & Model & $20.58 \pm 0.05$ & $20.37 \pm 0.05$ & $20.25 \pm 0.08$ & $20.35 \pm 0.32$ & SDSS \\
\hline SDSS DR15 & Host & Petrosian & $20.64 \pm 0.13$ & $20.40 \pm 0.09$ & $20.17 \pm 0.19$ & $\cdots$ & SDSS \\
\hline PS $13 \pi$ & Host & Kron & $21.39 \pm 0.08$ & $20.64 \pm 0.10$ & $20.48 \pm 0.06$ & $21.17 \pm 0.29$ & PS1 \\
\hline PS $13 \pi$ & Host & Aperture & $21.43 \pm 0.08$ & $20.62 \pm 0.08$ & $20.59 \pm 0.05$ & $21.07 \pm 0.17$ & PS1 \\
\hline
\end{tabular}

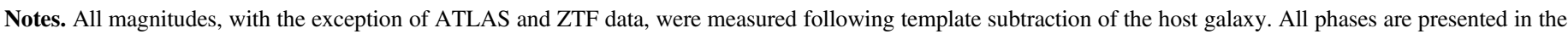
observer frame with respect to the ZTF discovery epoch, MJD 58480.422. ATLAS filter points are converted to $r$ in subsequent plots.

${ }^{a}$ Denotes that these are AB magnitudes in the ATLAS $o$ and $c$ filters respectively.

${ }^{\mathrm{b}}$ Denotes magnitudes obtained via aperture photometry, as opposed to PSF photometry, due to trailing in the input images.

radioactivity and energy from the spin-down of a magnetic neutron star. In addition we also compare our measured lightcurve to published models of rapidly evolving transients. Figure 3 shows the model comparisons, illustrating that the rapid decline rate cannot be fit with a radioactively powered model. To produce a peak luminosity of $L \sim 10^{43} \mathrm{erg} \mathrm{s}^{-1}$ a mass of $0.17 M_{\odot}$ is required if ${ }^{56} \mathrm{Ni}$ is the sole powering source:

$$
L_{56 \mathrm{Ni}}(t)=7.8 \times 10^{43}\left(\frac{M_{56 \mathrm{Ni}}}{1 M_{\odot}}\right) e^{-t / \tau_{56 \mathrm{Ni}}} \quad \operatorname{erg~s}^{-1} .
$$

Following Equation (1), semianalytical solutions for such a pure ${ }^{56} \mathrm{Ni}$ model are unable to adequately fit the decline rate as shown in Figure 3. We show our formal best-fit model for ${ }^{56} \mathrm{Ni}$ only powering which has an ejecta mass of $0.28 M_{\odot}$ assuming an opacity of $\kappa=0.1 \mathrm{~cm}^{2} \mathrm{~g}^{-1}$ and ${ }^{56} \mathrm{Ni}$ mass of $0.07 M_{\odot}$. Such an ejecta mass has a 5 day rise to peak, a blackbody temperature of $T_{\text {eff }} \sim 9000 \mathrm{~K}$ and would require a velocity of around $20,000-30,000 \mathrm{~km} \mathrm{~s}^{-1}$. This is simply the best fit to the data from a reduced $\chi^{2}$ statistic. Such a model could be scaled up to fit the peak with a significantly higher mass of ${ }^{56} \mathrm{Ni}$, but declines much too slowly to match the observed data.

The core collapse of an ultra-stripped He star model of Tauris et al. (2013) has been previously applied to rapidly declining transients such as SN2005ek (Drout et al. 2013). The progenitor transfers material to a compact companion and experiences iron core collapse while only just above the Chandrasekhar limit. As can be seen in Figure 3, even this ultra-stripped model, with $M_{\mathrm{ej}}=0.1 M_{\odot}$ and $M_{56 \mathrm{Ni}}=0.05 M_{\odot}$, 

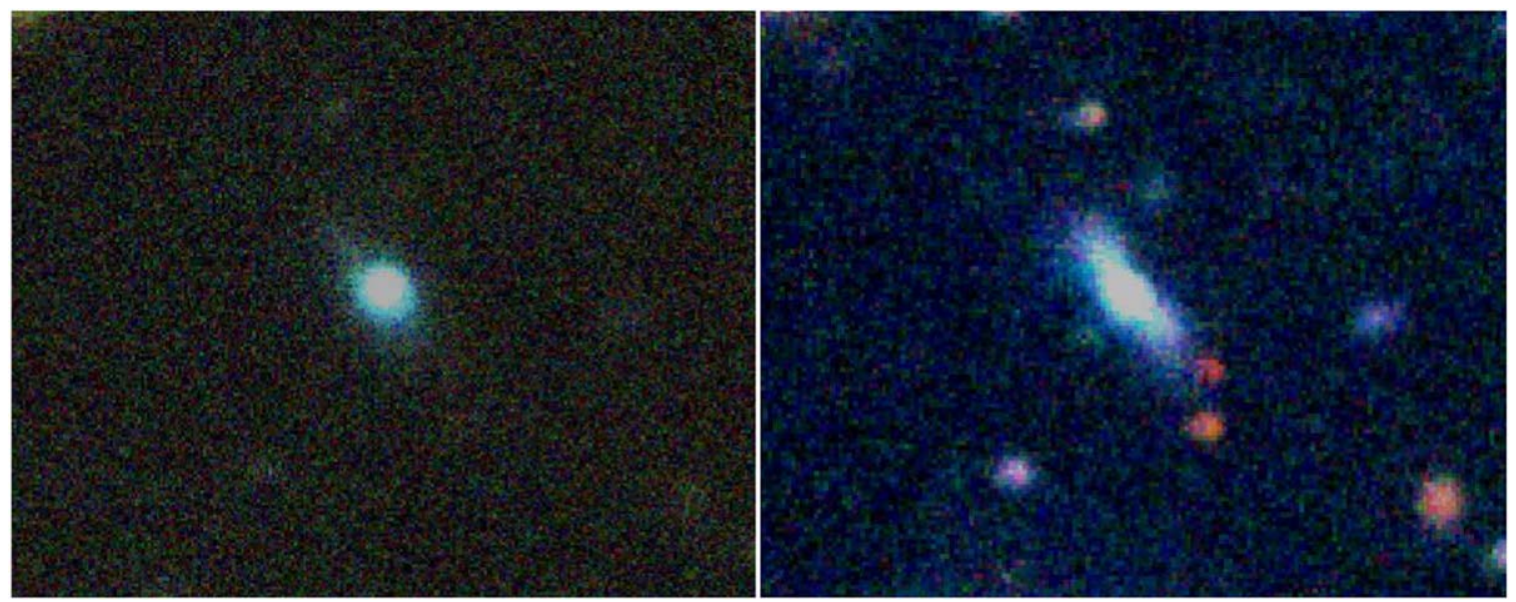

Figure 1. RGB composite images of the host of SN2018kzr, SDSS J082853.50+010638.6. Left: the GROND gri exposures from +3.731 days. Right: the NTT: EFOSC2 gri exposures taken at +68.676 days (Table 1 ). The host is a blue star-forming galaxy with a bright core.

Table 2

The Swift UVOT Photometric Log of SN2018kzr

\begin{tabular}{|c|c|c|c|c|c|c|c|c|}
\hline Date & MJD & Phase & $U V W 2$ & $U V M 2$ & $U V W 1$ & $U$ & $B$ & V \\
\hline 2019 Jan 1 15:54:43 & 58484.663 & 4.241 & $21.55 \pm 0.31$ & $21.31 \pm 0.35$ & $\cdots$ & $\cdots$ & $19.85 \pm 0.36$ & $19.41 \pm 0.49$ \\
\hline 2019 Jan 2 05:35:31 & 58485.233 & 4.811 & $\ldots$ & $\cdots$ & $\ldots$ & $21.20 \pm 0.33$ & $20.29 \pm 0.35$ & $\ldots$ \\
\hline 2019 Jan 2 17:48:28 & 58485.742 & 5.320 & $23.15 \pm 1.74$ & $\cdots$ & $21.77 \pm 0.34$ & $21.35 \pm 0.45$ & $21.34 \pm 1.19$ & $\ldots$ \\
\hline 2019 Jan 2 19:24:57 & 58485.809 & 5.387 & $\cdots$ & $\cdots$ & $21.73 \pm 0.34$ & $\cdots$ & $\ldots$ & $\cdots$ \\
\hline 2019 Jan 3 15:33:07 & 58486.648 & 6.226 & $\ldots$ & $21.79 \pm 0.26$ & $\ldots$ & $\cdots$ & $\ldots$ & $\ldots$ \\
\hline 2019 Jan 3 19:43:40 & 58486.822 & 6.400 & $22.14 \pm 0.19$ & $21.83 \pm 0.18$ & $21.83 \pm 0.21$ & $\cdots$ & $\cdots$ & $\cdots$ \\
\hline 2019 Jan 4 23:36:57 & 58487.984 & 7.562 & $\cdots$ & $\cdots$ & $\cdots$ & $\cdots$ & $\cdots$ & $\cdots$ \\
\hline 2019 Jan 5 22:53:45 & 58488.954 & 8.532 & $21.50 \pm 0.27$ & $\ldots$ & $\ldots$ & $\cdots$ & $\ldots$ & $\ldots$ \\
\hline
\end{tabular}

Note. These magnitudes are not host subtracted. All phases are presented in the observer frame with respect to the ZTF discovery epoch, MJD 58480.422.

does not decline rapidly enough to describe SN2018kzr. We discuss this explosion scenario in more depth in Section 4.

To further illustrate that rapidly declining models that are ${ }^{56} \mathrm{Ni}$ powered are inconsistent with the observed data, we show a set of thermonuclear explosion models for low mass carbonoxygen (CO) white dwarfs (WDs) from Sim et al. (2012) in the right panel of Figure 3. The Sim et al. (2012) models have a $\mathrm{CO}$ core that accretes a sufficiently large helium layer prior to the ignition of core nuclear burning such that the He layer itself instigates a detonation. This primary detonation extends into the $\mathrm{CO}$ core wherein a secondary detonation may occur-the Edge-Lit Double Detonation scenario. The primary detonation may, however, be the only detonation to occur, giving the Helayer Detonation scenario. Two sets of models are presented for each scenario, one being the nominated standard system (Model S) with a core mass of $M_{\mathrm{CO}}=0.58 M_{\odot}$ and envelope mass of $M_{\mathrm{He}}=0.21 M_{\odot}$, and another being a specific low mass system (Model L) where the core mass is reduced to $M_{\mathrm{CO}}=0.45 M_{\odot}$. The helium shell detonation models (.Ia models) of Shen et al. (2010) are also either too faint, too slowly evolving or too red (see Section 3.2) to be viable explanations. From this, we disfavor a low mass, thermonuclear explosion, or any type of radioactively powered explosion where the dominant component is ${ }^{56} \mathrm{Ni}$ as the explosion scenario for SN2018kzr. We also disfavor powering from other radioactive isotopes, such as ${ }^{48} \mathrm{Cr}$ or ${ }^{52} \mathrm{Fe}$, which may have a shorter lifetime than ${ }^{56} \mathrm{Ni}$ (Dessart et al. 2014). The energy release per unit mass from the decay of these isotopes is notably lower than that of ${ }^{56} \mathrm{Ni}$ which would necessitate a larger quantity of each to be synthesized compared to the amount of ${ }^{56} \mathrm{Ni}$ synthesized in order to explain the lightcurve evolution of this object.

An extra powering source is therefore required, and hence we move to testing a model with additional energy from a central engine. We employ a magnetar spin-down component as conceived by Kasen \& Bildsten (2010) and Woosley (2010) and further generalized for lightcurve fitting by Inserra et al. (2013). ${ }^{25}$ Our model supplements ${ }^{56} \mathrm{Ni}$ decay with powering from the magnetar's rotational kinetic energy as it spins down. These models assume an explosion energy of $10^{51} \mathrm{erg}$, a magnetar radiation opacity of $0.01 \mathrm{~cm}^{2} \mathrm{~g}^{-1}$ and an electromagnetic radiation opacity of $0.1 \mathrm{~cm}^{2} \mathrm{~g}^{-1}$. We choose this latter opacity as it is within the limit allowed for electron scattering, assuming it is not influenced by line contributions. We first considered only the magnetar spin-down component in the absence of ${ }^{56} \mathrm{Ni}$ powering and found a reasonable fit which implied an ejecta mass of $0.1 M_{\odot}$, along with an initial magnetar spin period of $P=25 \mathrm{~ms}$ and magnetic field of $B=25 \times 10^{14} \mathrm{G}$. This fit is shown in Figure 3. In general, it adequately describes the rapid decline of SN2018kzr but falls below the luminosity of the final data point on the lightcurve. For these fit parameters the spin-down timescale for a magnetar would be approximately 7 days. Hence, given the lifetime of SN2018kzr the input magnetar energy would only decline by a factor of a few. However, the output magnetar energy declines

$\overline{25}$ https://bitbucket.org/andersjerkstrand/lcmodels/src/master/ 
Table 3

The Spectroscopic Log of SN2018kzr

\begin{tabular}{|c|c|c|c|c|c|c|}
\hline Date & MJD & Phase & Telescope & Instrument & Spectral Range & Spectral Resolution \\
\hline 2018 Dec 30 07:36:05 & 58482.317 & 1.895 & NTT & EFOSC2 & $3700-9300 \AA$ & 355 \\
\hline 2018 Dec 31 05:56:13 & 58483.247 & 2.825 & NTT & EFOSC2 & $3700-9300 \AA$ & 355 \\
\hline 2019 Jan 1 04:06:59 & 58484.172 & 3.750 & NTT & EFOSC2 & 3400-10300 A & $390^{\mathrm{a}}, 595^{\mathrm{b}}$ \\
\hline 2019 Jan 4 05:45:10 & 58487.240 & $6.818^{\mathrm{c}}$ & VLT & Xshooter & 3100-10300 A & 3300 \\
\hline 2019 Jan 4 08:19:41 & 58487.347 & 6.925 & Gemini & GMOS-N & $4200-9000 \AA$ & 1918 \\
\hline 2019 Jan 4 11:27:55 & 58487.478 & 7.056 & Keck & LRIS & 3000-10300 A & 1050 \\
\hline 2019 Jan 4 15:17:01 & 58487.637 & 7.215 & SALT & RSS & 3600-8300 А & 1277 \\
\hline 2019 Jan 6 10:29:17 & 58489.437 & $9.015^{\mathrm{c}}$ & Gemini & GMOS-N & $4200-9000 \AA$ & 1918 \\
\hline 2019 Jan 7 07:35:21 & 58490.316 & $9.894^{\mathrm{c}}$ & NTT & EFOSC2 & $3700-9300 \AA$ & 355 \\
\hline 2019 Jan 11 08:33:05 & 58494.356 & 14.136 & Keck & LRIS & 3000-10300 A & 1050 \\
\hline 2019 Feb1 1 02:50:46 & 58525.119 & 44.697 & VLT & Xshooter & 3700-20700 A & 3300 \\
\hline
\end{tabular}

Notes. All phases are presented in the observer frame with respect to the ZTF discovery epoch, MJD 58480.422.

a Denotes the resolution is for the Grism\#11 with EFOSC2.

${ }^{\mathrm{b}}$ Denotes the resolution is for the Grism\#16 with EFOSC2.

${ }^{c}$ Denotes the spectra are of low signal, being observed either in poor conditions or with the transient not centered in the slit.



Figure 2. Combined ZTF, ATLAS, GROND, LT, and NTT lightcurves compared to the compiled lightcurves of two other notable fast transientsthose being AT2018cow (Prentice et al. 2018) and the kilonova, AT2017gfo (Andreoni et al. 2017; Arcavi et al. 2017; Chornock et al. 2017; Cowperthwaite et al. 2017; Drout et al. 2017; Evans et al. 2017; Kasliwal et al. 2017; Lipunov et al. 2017; Nicholl et al. 2017; Pian et al. 2017; Smartt et al. 2017; Tanvir et al. 2017; Troja et al. 2017; Utsumi et al. 2017; Valenti et al. 2017).

by a factor of nearly 100 in this time, implying the rapid evolution is driven by declining trapping of the magnetar radiation.

It is possible to add a small quantity of ${ }^{56} \mathrm{Ni}$ to the magnetar model to slow the decline in the tail of the lightcurve and enable a better fit to the late lightcurve. We observe that $0.02 M_{\odot}$ of ${ }^{56} \mathrm{Ni}$ is required to cause a noticeable change in the fit profile, but that this is insufficient for the fit to encompass the final data point. Further increases in ${ }^{56} \mathrm{Ni}$ produce less physically plausible fits as the ${ }^{56} \mathrm{Ni}$ fraction begins to tend to unity. Furthermore, the bolometric luminosity at this point is uncertain by 0.2 dex. Scaling the +14.136 days Keck:LRIS spectrum to the gri photometry at +16.792 days and integrating the spectral flux gives a luminosity $\log L_{\mathrm{bol}}=$ 40.7 dex indicating the data may not be significantly discrepant from the model. Both the magnetar only and magnetar supplemented by ${ }^{56} \mathrm{Ni}$ heating models favor an ejecta temperature in the range of $16,000-18,000 \mathrm{~K}$ and photospheric velocity of $\sim 0.1 c$ at time when the bolometric lightcurve is at peak.

\subsection{Spectral Analysis and Modeling}

Our early spectra were modeled with TARDIS (Kerzendorf \& Sim 2014) and a model fit is shown in Figure 4 for the NTT: EFOSC $2+2.825$ days spectrum. There are four strong absorption features with minima at 3900,4300, 5000, and $6100 \AA$ which are reproduced in our model by Ca II, Fe II, and Si II, with a model velocity of $\sim 12,000 \mathrm{~km} \mathrm{~s}^{-1}$.

The model is primarily composed of $\mathrm{O}(\sim 75 \%)$, with significant amounts of intermediate mass elements, primarily $\mathrm{Si}$ and $\mathrm{Mg}$ ( $\sim 10 \%$ each), along with some Fe-group elements. To reproduce the $\mathrm{Fe}$ II features in our observed spectra we require $\sim 3 \%$ of the total ejecta mass to be $\mathrm{Fe}$ in our model. We previously found that $20 \%$ of the ejecta being ${ }^{56} \mathrm{Ni}$ is required to impact the lightcurve fit, but for this composition if as much of the $5 \%-10 \%$ of the ejecta is ${ }^{56} \mathrm{Ni}$ it begins to present significantly in the spectral model fit. Hence, we disfavor a large amount of ${ }^{56} \mathrm{Ni}$ in the ejecta.

The temperature, ejecta mass, and luminosity required for the spectral fit in Figure 4 are consistent with the lightcurve model, with some minor discrepancies. The model spectrum is 7 days after explosion, whereas the lightcurve fit implies this spectrum should be $4-5$ days after explosion. This may imply the ejecta is not in homologous expansion and given the simplicity of our magnetar model for the lightcurve, where the hydrodynamics of the pulsar wind bubble is not numerically modeled, we do not consider this a serious physical inconsistency. The ejecta velocity implied by the lightcurve modeling stands at a factor of three greater than that by the spectral modeling. This is likely the result of a longer rise time than is assumed by the lightcurve model, a non-homologous expansion of material or the ejecta being non-spherical. Further quantitative modeling of all spectra and a more detailed description of the radiative transfer will be presented in a companion paper (J. H. Gillanders et al. 2019, in preparation). 


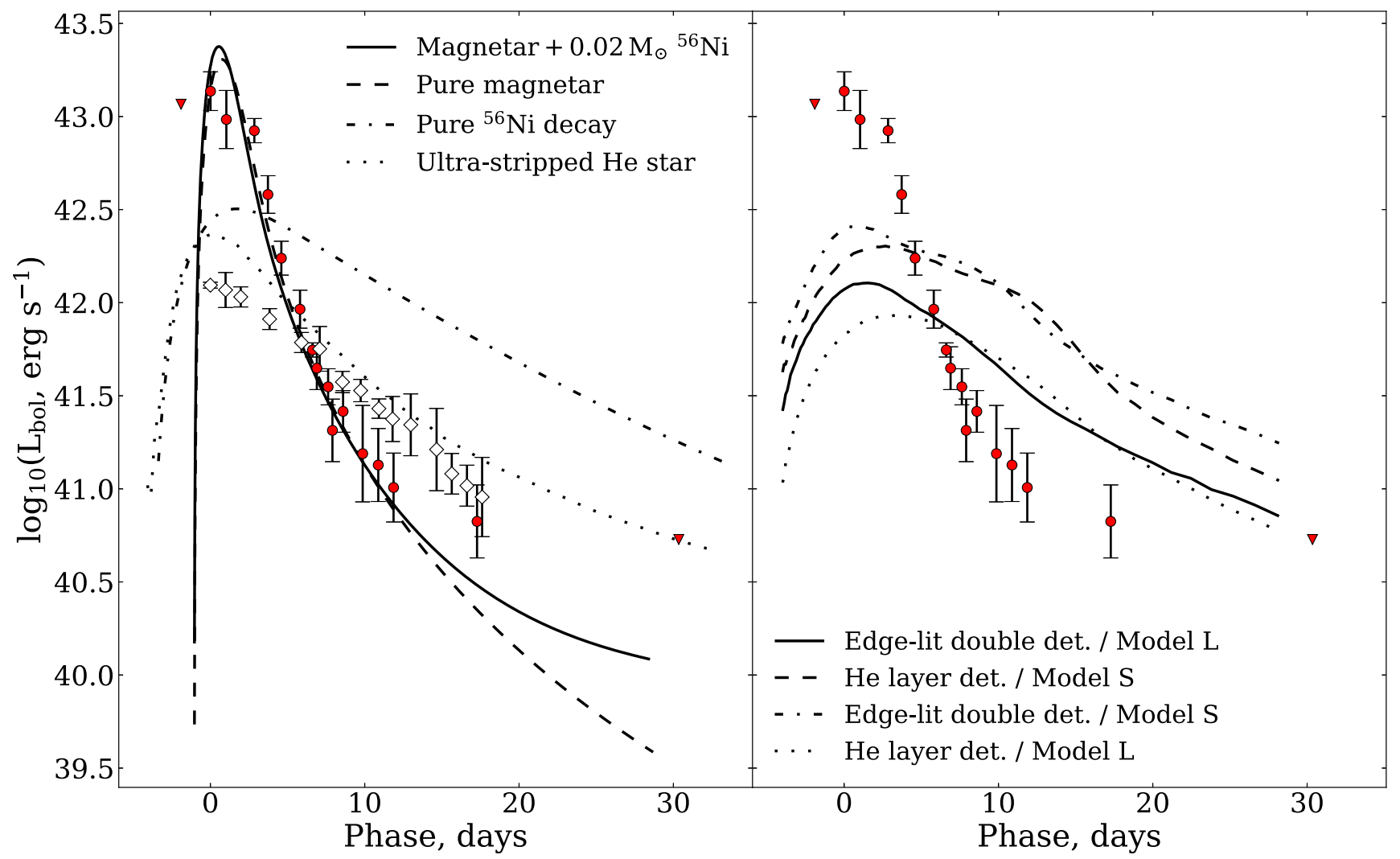

Figure 3. Left: the bolometric lightcurve of SN2018kzr along with several model fits of the powering source including pure ${ }^{56} \mathrm{Ni}$, a mix of ${ }^{56} \mathrm{Ni}$ heating and magnetar spin-down and the explosion of a stripped He star (Tauris et al. 2013), with the bolometric lightcurve of SN2005ek (Drout et al. 2013) for which this model was developed. Right panel: the bolometric lightcurve of SN2018kzr in comparison to several fast evolving thermonuclear progenitor models detailed in Sim et al. (2012).

In Figure 5, we show the two spectra taken at approximately +7 days from Gemini:GMOS-N and Keck:LRIS (see Table 3) as well as the Keck:LRIS spectrum from +14.136 days. As the transient faded rapidly, contamination from the host galaxy became significant at these epochs. The VLT:Xshooter spectrum from +44.697 days, which is purely host flux, was smoothed and subtracted from the spectra. The spectra were scaled and subtracted such that the final host subtracted spectra matched the difference image photometry through synthetic photometry in the riz bands. The flux levels are not reliable below $4500 \AA$ due to the strong host flux at these wavelengths. There is a remarkable similarity between the Keck:LRIS +7.056 days spectrum of SN2018kzr and a SN2018byg spectrum taken +27 days from its $r$ band maximum. SN2018byg, discussed by De et al. (2019), has been presented as the result of a double detonation of a CO WD surrounded by a He shell, much like the models presented by Sim et al. (2012). The spectra around peak for SN2018byg are noted by De et al. (2019) to show line blanketing from Fe-group elements indicative of a large $\mathrm{Fe}$ mass in the outermost layers of the ejecta. The features at 4500 and $5500 \AA$ are weaker in the spectrum of SN2018kzr. De et al. (2018) attribute the features to Ca II and Ti II in SN2018byg. At this stage, SN2018kzr is entering the nebular phase and further analysis of the ionic species producing these features will be discussed in Gillanders et al. (2019, in preparation).

By +14 days, the 5500 and $6500 \AA$ features have disappeared, leaving a strong and broad feature centered on $8450 \AA$. The obvious candidate is the Ca II triplet; however, the centroid of the feature is $\sim 120 \AA\left(\sim 4200 \mathrm{~km} \mathrm{~s}^{-1}\right)$ offset from its rest wavelength position.

\section{Explosion Mechanism and Scenario}

Our data show SN2018kzr is the fastest declining supernovalike transient apart from the kilonova, AT2017gfo. We rule out an NS-NS merger for SN2018kzr due to the TARDIS spectroscopic model composition which is predominantly intermediate mass elements including $\mathrm{O}, \mathrm{Mg}, \mathrm{Si}$, and $\mathrm{Ca}$, along with a small fraction of $\mathrm{Fe}$. The lightcurve and spectra cannot be explained only through radioactive powering by ${ }^{56} \mathrm{Ni}$ and we instead favor a magnetar powering mechanism. This powering mechanism provides a model which is quantitatively a good fit to the data with an ejecta mass of $M_{\mathrm{ej}} \simeq 0.1 M_{\odot}$, and a neutron star with period $P \simeq 25 \mathrm{~ms}$ and magnetic field of $B \simeq 25 \times 10^{14} \mathrm{G}$. We disfavor any He-detonation or thermonuclear model due to the fast and luminous lightcurve, which is physically inconsistent with ${ }^{56} \mathrm{Ni}$ powering. Three possible progenitor scenarios and explosion mechanisms are worth considering that have previously been investigated and predict low mass ejecta with intermediate mass elements and an alternative power source to radioactive decay. These are an ultra-stripped core collapse of a massive star, accretion induced collapse (AIC) of an oxygen-neon (ONe) WD, and a WD-NS merger.

Ultra-stripped core collapse model: The ultra-stripped core collapse model has a He star with total mass before explosion which is only just above the Chandrasekhar limit (e.g., 0.05-0.20 $M_{\odot}$, Tauris et al. 2015), due to mass-loss from a common envelope phase and accretion onto a neutron star companion in a tight orbit. The models of Tauris et al. (2013) have successfully reproduced rapidly declining transients such as SN2005ek (Drout et al. 2013, which we show in the left 


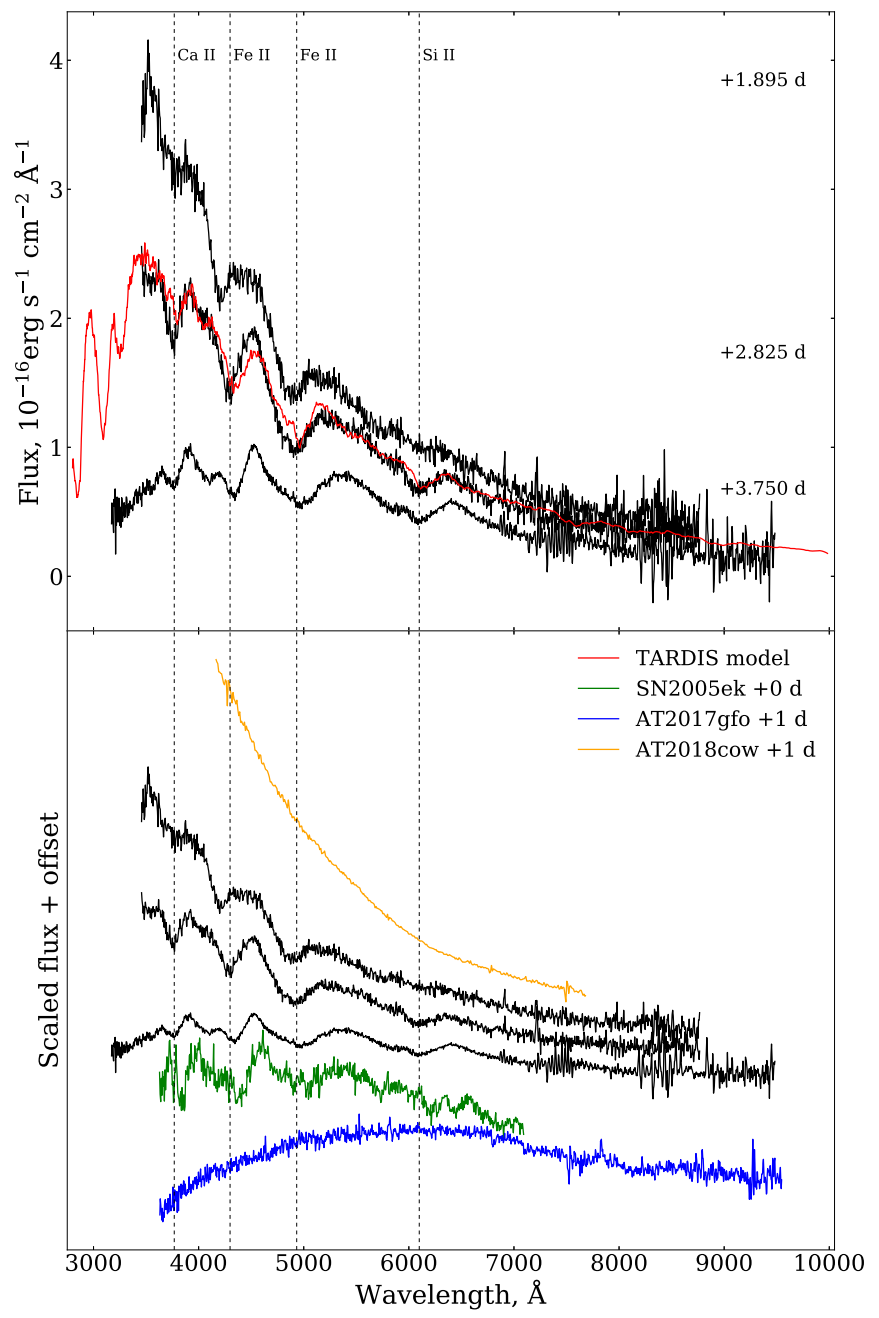

Figure 4. Top: the early spectral sequence of SN2018kzr comprised of the first three NTT spectra with phases shown relative to the ZTF discovery epoch Overlaid in red on the +2.825 days spectrum is a TARDIS (Kerzendorf \& Sim 2014) model investigating the composition of the progenitor. Bottom: the early spectral sequence of SN2018kzr along with several comparison spectra of fast transients including SN2005ek (Drout et al. 2013), AT2017gfo (Smartt et al. 2017), and AT2018cow (Prentice et al. 2018). Phases of the comparison spectra are given with respect to the object's maximum light. The spectra have been dereddened and corrected for redshift.

panel of Figure 3 for illustrative purposes). However, such an explosion scenario is unlikely to produce as rapidly rotating a remnant as we present here. For a given He star, the largest component of angular momentum will be held in the envelope rather than the core. Rapid stripping of the envelope via mass transfer to a compact companion does not normally facilitate redistribution of angular momentum to the core. This is supported by multi-dimensional simulations of Müller et al. (2018) which lead to slowly spinning progenitors, far from the $25 \mathrm{~ms}$ rotation rate required to provide the observed luminosity.

Accretion induced collapse of a white dwarf: The AIC of an ONe WD has been predicted to lead to a rapidly rotating neutron star in which magnetic fields may be large (up to $10^{15} \mathrm{G}$; Dessart et al. 2007). These simulations predict a magnetically enhanced explosion leaving behind a rapidly rotating millisecond pulsar, along with an ejection of $\sim 0.1 M_{\odot}$ of material with only traces of ${ }^{56} \mathrm{Ni}$. The He star + ONe WD binary simulations of Brooks et al. (2017) show that the

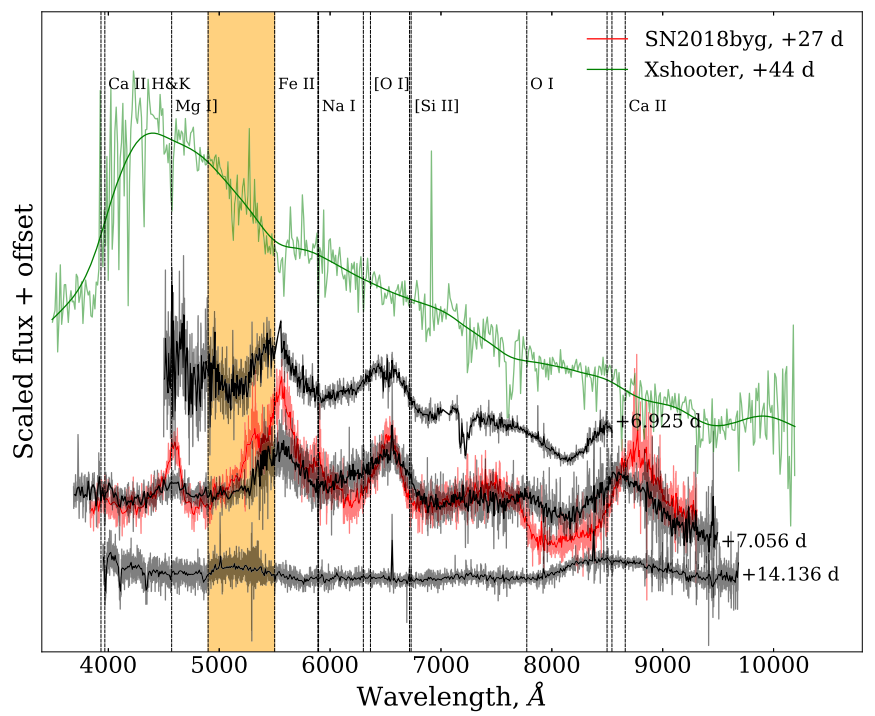

Figure 5. Gemini:GMOS-N spectrum and two Keck:LRIS spectra (black). The final VLT:Xshooter spectrum at +44.697 days has been heavily smoothed (green) and subtracted from these spectra to remove host contamination. The spectra have been rebinned to approximate $5 \AA$ per pixel resolution. Overlaid on the Keck:LRIS +7.056 days spectrum is a spectrum of SN2018byg at +27 days (red; De et al. 2019) from its $r$ band peak highlighting the similarities between these two objects despite their significantly different evolutionary timescales. The shaded region indicates the wavelength range over which Fe II emission occurs.

accretion from a He star companion can lead to an outer layer structure on the $\mathrm{ONe} W D$ which is composed of $\mathrm{O}, \mathrm{Ne}, \mathrm{Si}$, and $\mathrm{Mg}$. The 1.0-1.3 $M_{\odot} \mathrm{WD}$ grows, reaching close to the Chandrasekhar limit, which triggers electron capture in the core resulting in AIC. The composition of the WD calculated by Brooks et al. (2017) is compatible with our estimates from the spectral models. However, several simulations have predicted significantly heavier elements should characterize the ejecta of AIC events. Metzger et al. (2009) and Darbha et al. (2010) predict a composition rich in Fe-group elements, while the Dessart et al. (2007) simulations produce ejecta with a low electron fraction and a composition dominated by elements heavier than Fe. It appears none of these models produce the intermediate mass element composition apparent in our spectra.

White dwarf-neutron star mergers: The WD-NS merger scenario involves the production of an accretion disk following the tidal disruption of a sufficiently massive WD as it inspirals with an NS companion. The disk will be comprised of WD material, provided the WD has mass $\gtrsim 0.65 M_{\odot}$ (Margalit \& Metzger 2016), and the temperature and midplane density are predicted to be high enough to support burning of WD material to higher mass elements (Metzger 2012). For a CO WD, the ejecta may contain the intermediate mass elements observed in SN2018kzr (O, Si, and $\mathrm{Mg}$ ) in addition to $10^{-3}-10^{-2} M_{\odot}$ of ${ }^{56} \mathrm{Ni}$ (Metzger 2012). This ${ }^{56} \mathrm{Ni}$ can only power a faint optical transient of peak luminosity $\sim 10^{40} \mathrm{erg} \mathrm{s}^{-1}$. However, high velocity winds from the disk can produce shocks which thermalize the kinetic energy of the winds to power characteristic luminosities of $10^{43} \mathrm{erg} \mathrm{s}^{-1}$ (Margalit \& Metzger 2016). Interestingly the timescale of the powering falls off as $\dot{E} \sim t^{-5 / 3}$. This is similar to the magnetar powering function $\left(t^{-2}\right)$ and hence would likely result in a similar lightcurve. Schwab et al. (2016) suggest that another possible channel to produce a rapidly rotating neutron star remnant is through the 
merger of two WDs, potentially avoiding thermonuclear runaway and creating a massive, rapidly rotating WD that will likely collapse (Gvaramadze et al. 2019).

Of these scenarios, we disfavor the ultra-stripped core collapse scenario, owing predominantly to the fact that it would not accommodate such a rapidly rotating neutron star as we are suggesting here. We instead favor the AIC or WD-NS merger scenarios as they are consistent with an ejecta mass of $M_{\mathrm{ej}}=0.1 \pm 0.05 M_{\odot}$ and the requirement from our bolometric lightcurve modeling that the powering mechanism be supplemented by an additional component, likely a rapidly rotating magnetar. Our spectral modeling indicates a composition of primarily intermediate mass elements. In the case of AIC, it is unlikely that this would be observed based on current models (Dessart et al. 2007; Metzger et al. 2009; Darbha et al. 2010); however, such a composition is plausible for a WD-NS merger (Metzger 2012).

Based in part on observations collected at the European Organisation for Astronomical Research in the Southern Hemisphere, Chile as part of the extended Public ESO Spectroscopic Survey for Transient Objects (ePESSTO), program 199.D-0143, the SALT Large Science Programme on transients (2018-2-LSP001), MNiSW DIR/WK/2016/07, GROND support through DFG grant HA $1850 / 28-1$. ATLAS is supported primarily through NASA grant NN12AR55G, 80NSSC18K1575. This research made use of TARDIS supported by the Google Summer of Code, ESA's Summer of Code in Space program. Funding acknowledgments: STFC ST/P000312/1 (SJS, SAS); Alexander von Humboldt Foundation (TWC); ERC and H2020 MSC grants [615929, 725161, 758638] (A.G.Y., C.P.G., K.M., and L.G.); ISF GW excellence center, IMOS, BSF Transformative program, Benoziyo Endowment Fund for the Advancement of Science, Deloro Institute, Veronika A. Rabl Physics Discretionary Fund, Paul and Tina Gardner and the WIS-CIT, Helen and Martin Kimmel Award (AGY); RAS Research Fellowship (MN), Polish NCN MAESTRO grant 2014/14/A/ST9/00121 (MG), IC120009 "Millennium Institute of Astrophysics" of the Iniciativa Científica Milenio del Ministerio Economía, Fomento y Turismo de Chile and CONICYT PAI/INDUSTRIA 79090016 (OR); NRF South Africa (DAHB) LASAIR is supported by STFC grants ST/N002512/1 and ST/N002520/1.

Facilities: ESO (NTT, VLT), Gemini (GMOS), Keck (LRIS), SALT (RSS), Swift (XRT and UVOT), SALT, Max Planck:2.2m, ATLAS, ZTF, Liverpool:2m (IO:O), LCO.

\section{ORCID iDs}

Owen R. McBrien (iD https://orcid.org/0000-0001-8049-4036 Stephen J. Smartt (D) https://orcid.org/0000-0002-8229-1731 Ting-Wan Chen (ib https://orcid.org/0000-0003-1532-0149 Cosimo Inserra (1D https://orcid.org/0000-0002-3968-4409 Stuart A. Sim (iD https://orcid.org/0000-0002-9774-1192 Anders Jerkstrand (i) https://orcid.org/0000-0001-8005-4030 Stefano Valenti (1D https://orcid.org/0000-0001-8818-0795 Rupak Roy (1) https://orcid.org/0000-0002-9711-6207 Stefan Taubenberger (iD https://orcid.org/0000-00024265-1958

Mark E. Huber (iD https://orcid.org/0000-0003-1059-9603 Ken C. Chambers (i) https://orcid.org/0000-0001-6965-7789 Avishay Gal-Yam (ib https://orcid.org/0000-0002-3653-5598 David R. Young (i) https://orcid.org/0000-0002-1229-2499 Matt Nicholl (i) https://orcid.org/0000-0002-2555-3192
Ken W. Smith (1D https://orcid.org/0000-0001-9535-3199

Ilya Mandel (i) https://orcid.org/0000-0002-6134-8946

Simon Prentice (1) https://orcid.org/0000-0003-0486-6242

Ósmar Rodríguez (D) https://orcid.org/0000-0001-8651-8772

Claudia P. Gutiérrez (i) https://orcid.org/0000-0003-

2375-2064

Lluís Galbany (iD https://orcid.org/0000-0002-1296-6887

Jesper Sollerman (1D https://orcid.org/0000-0003-1546-6615

Shrinivas R. Kulkarni (iD https://orcid.org/0000-0001-

5390-8563

Arne Rau (iD https://orcid.org/0000-0001-5990-6243

\section{References}

Abbott, B. P., Abbott, R., Abbott, T. D., et al. 2017, PhRvL, 119, 161101 Andreoni, I., Ackley, K., Cooke, J., et al. 2017, PASA, 34, e069

Arcavi, I., Hosseinzadeh, G., Howell, D. A., et al. 2017, Natur, 551, 64 Becker, A. 2015, HOTPANTS: High Order Transform of PSF ANd Template Subtraction, Astrophysics Source Code Library, ascl:1504.004

Bellm, E. C., Kulkarni, S. R., Graham, M. J., et al. 2019, PASP, 131, 018002

Brooks, J., Schwab, J., Bildsten, L., Quataert, E., \& Paxton, B. 2017, ApJ, 843, 151

Cardelli, J. A., Clayton, G. C., \& Mathis, J. S. 1989, ApJ, 345, 245

Chambers, K. C., Magnier, E. A., Metcalfe, N., et al. 2016, arXiv:1612.05560

Chen, P., Dong, S., Stritzinger, M. D., et al. 2019, arXiv:1905.02205

Chen, T. W., Inserra, C., Fraser, M., et al. 2018, ApJL, 867, L31

Chornock, R., Berger, E., Kasen, D., et al. 2017, ApJL, 848, L19

Coulter, D. A., Foley, R. J., Kilpatrick, C. D., et al. 2017, Sci, 358, 1556

Cowperthwaite, P. S., Berger, E., Villar, V. A., et al. 2017, ApJL, 848, L17

Darbha, S., Metzger, B. D., Quataert, E., et al. 2010, MNRAS, 409, 846

De, K., Kasliwal, M. M., Ofek, E. O., et al. 2018, Sci, 362, 201

De, K., Kasliwal, M. M., Polin, A., et al. 2019, ApJL, 873, L18

Dessart, L., Burrows, A., Livne, E., \& Ott, C. D. 2007, ApJ, 669, 585

Dessart, L., Hillier, D. J., Blondin, S., \& Khokhlov, A. 2014, MNRAS, 441, 3249

Drout, M. R., Piro, A. L., Shappee, B. J., et al. 2017, Sci, 358, 1570

Drout, M. R., Soderberg, A. M., Mazzali, P. A., et al. 2013, ApJ, 774, 58

Evans, P. A., Cenko, S. B., Kennea, J. A., et al. 2017, Sci, 358, 1565

Fremling, C. 2018, TNSTR, 1996

Greiner, J., Bornemann, W., Clemens, C., et al. 2008, PASP, 120, 405

Gvaramadze, V. V., Gräfener, G., Langer, N., et al. 2019, Natur, 569, 684

Inserra, C., Smartt, S. J., Jerkstrand, A., et al. 2013, ApJ, 770, 128

Kasen, D., \& Bildsten, L. 2010, ApJ, 717, 245

Kasen, D., Metzger, B., Barnes, J., Quataert, E., \& Ramirez-Ruiz, E. 2017, Natur, 551, 80

Kasliwal, M. M., Kulkarni, S. R., Gal-Yam, A., et al. 2010, ApJL, 723, L98

Kasliwal, M. M., Nakar, E., Singer, L. P., et al. 2017, Sci, 358, 1559

Kerzendorf, W. E., \& Sim, S. A. 2014, MNRAS, 440, 387

Li, W., Filippenko, A. V., Chornock, R., et al. 2003, PASP, 115, 453

Lipunov, V. M., Gorbovskoy, E., Kornilov, V. G., et al. 2017, ApJL, 850, L1

Magnier, E. A., Schlafly, E. F., Finkbeiner, D. P., et al. 2016, arXiv:1612. 05242

Margalit, B., \& Metzger, B. D. 2016, MNRAS, 461, 1154

Metzger, B. D. 2012, MNRAS, 419, 827

Metzger, B. D. 2017, LRR, 20, 3

Metzger, B. D., Piro, A. L., \& Quataert, E. 2009, MNRAS, 396, 1659

Müller, B., Gay, D. W., Heger, A., Tauris, T. M., \& Sim, S. A. 2018, MNRAS, 479, 3675

Nicholl, M. 2018, RNAAS, 2, 230

Nicholl, M., Berger, E., Kasen, D., et al. 2017, ApJL, 848, L18

Perets, H. B., Gal-Yam, A., Mazzali, P. A., et al. 2010, Natur, 465, 322

Pian, E., D’Avanzo, P., Benetti, S., et al. 2017, Natur, 551, 67

Pineda, J., Razza, A., Gromadzki, M., et al. 2018, ATel, 12347

Poznanski, D., Chornock, R., Nugent, P. E., et al. 2010, Sci, 327, 58

Prentice, S. J., Maguire, K., Smartt, S. J., et al. 2018, ApJL, 865, L3

Razza, A., Pineda, J., Gromadzki, M., et al. 2018, ATel, 12342

Rest, A., Garnavich, P. M., Khatami, D., et al. 2018, NatAs, 2, 307

Schwab, J., Quataert, E., \& Kasen, D. 2016, MNRAS, 463, 3461

Shen, K. J., Kasen, D., Weinberg, N. N., Bildsten, L., \& Scannapieco, E. 2010, ApJ, 715, 767

Sim, S. A., Fink, M., Kromer, M., et al. 2012, MNRAS, 420, 3003

Smartt, S. J., Chen, T.-W., Jerkstrand, A., et al. 2017, Natur, 551, 75

Smartt, S. J., Valenti, S., Fraser, M., et al. 2015, A\&A, 579, A40 
Smith, K. W., Williams, R. D., Young, D. R., et al. 2019, RNAAS, 3, 26 Tanvir, N. R., Levan, A. J., González-Fernández, C., et al. 2017, ApJL, 848, L27

Tauris, T. M., Langer, N., Moriya, T. J., et al. 2013, ApJL, 778, L23

Tauris, T. M., Langer, N., \& Podsiadlowski, P. 2015, MNRAS, 451, 2123

Tonry, J. L., Denneau, L., Heinze, A. N., et al. 2018, PASP, 130, 064505
Troja, E., Piro, L., van Eerten, H., et al. 2017, Natur, 551, 71

Utsumi, Y., Tanaka, M., Tominaga, N., et al. 2017, PASJ, 69, 101

Valenti, S., David, Sand, J., et al. 2017, ApJL, 848, L24

Valenti, S., Yuan, F., Taubenberger, S., et al. 2014, MNRAS, 437, 1519

Whitesides, L., Lunnan, R., Kasliwal, M. M., et al. 2017, ApJ, 851, 107

Woosley, S. E. 2010, ApJL, 719, L204 